\title{
Absorption coefficient and exposure kinetics of photoresists at EUV
}

\author{
Roberto Fallica $^{* a}$, Jarich Haitjema ${ }^{\mathrm{b}}$, Lianjia Wu ${ }^{\mathrm{b}}$, Sonia Castellanos ${ }^{\mathrm{b}}$, Fred Brouwer ${ }^{\mathrm{b}}$, Yasin Ekinci ${ }^{\mathrm{a}}$ \\ ${ }^{a}$ Paul Scherrer Institute, 5232 Villigen PSI, Switzerland \\ ${ }^{\mathrm{b}}$ Advanced Research Center for Nanolithography, Science Park 110, 1098XG Amsterdam, The Netherlands
}

\begin{abstract}
The experimental measurement of the time-dependent absorption of photoresists at extreme ultraviolet wavelength is of great interest for the modeling of the lithographic process. So far, several technical challenges have made the accurate determination of the linear absorption coefficient and the Dill parameters nontrivial. In this work, we use a dedicated equipment and synchrotron light source to experimentally measure the transmittance of thin layers of photoresists on transparent silicon nitride membranes, and their thickness was measured with the spectroscopic ellipsometry. The absorption of negative tone photocondensed metal oxide photoresists based on $\mathrm{Sn}$ cage structures, and of $\mathrm{Zr}$ and $\mathrm{Hf}$ oxoclusters was measured and compared to the estimated values. It was found that tin based materials absorb considerably more light than conventional chemically amplified resists based on organic polymer. Hafnium-based materials have about twice absorption, while zirconium based are basically comparable to organic resists. Furthermore, the exposure kinetics of several chemically amplified resists with varying photoacid concentration and backbone polymer was studied. The rate of bleaching, described by the Dill parameter $\mathrm{C}$, was measured and conclusions are drawn based on the specific resist formulation.
\end{abstract}

Keywords: EUV, absorption coefficient, Dill Parameter, exposure kinetics, quantum yield, tin cage, oxocluster, CAR

\section{INTRODUCTION}

In a photolithographic process, the amount of energy delivered in a homogeneous photoresist film depends on its thickness and the penetration depth of light in matter. According to the Beer-Lambert law, absorption is fully described by the linear absorption coefficient, $\alpha$. The absorption does not stay constant during exposure, but decreases as the resist becomes more transparent - an effect also known as bleaching. Assessing the magnitude of bleaching is crucial to the modeling and prediction of the photolithographic process. A simple and elegant description and methodology for the measurement of photoresist bleaching was introduced ${ }^{1}$ several decades ago. The three Dill parameters fully describe the dynamic behavior of resist and, over the course of the years, their widespread adoption testified to their success. Besides the technological interest, the measurement of the intrinsic physical quantity has a fundamental significance in materials science.

Photoresists for visible and i-line photolithography were based mostly on DNQ and novolak resins compounds. These materials were designed to coat at several hundreds of nanometers. Later, deep ultraviolet (DUV) photolithography has been using chemically amplified resist (CAR), based on polymer backbone. At those wavelength, these materials have $\alpha$ of the order of $\approx 1 \mu \mathrm{m}^{-1}$.

Extreme ultraviolet light (EUV, $\lambda=13.5 \mathrm{~nm}$ ) is considered as the most likely candidate for next generation lithography. The photochemistry of resist exposure at this wavelength is radically different ${ }^{2}$, and new insight needs to be gained by manufacturers and lithographers to improve the performance of EUV resists. In this shifting landscape, photoresist materials need to adjust their functionality to the new conditions. Specifically, because chemically amplification might be an unsurmountable limit to the achievement of the high resolution that EUV lithography promises, completely novel approaches are being explored. Among these, hybrid organometallic photoresists ${ }^{3}$, oxide nanoparticles ${ }^{4,5}$, and photocondensed molecular oxide ${ }^{6}$ testify to the intense scientific research in this field. In particular, photocondensed metal oxides have proven a remarkable combination of features (high resolution, high etch resistance, compatibility with existing process) that make them interesting for EUV lithography.

* roberto.fallica@psi.ch; phone 004156310 4578; fax 004156310 2111; www.psi.ch 
The inclusion of metallic elements and/or high $Z$-number elements is a novelty in photoresist synthesis. In the specific case of EUV lithography, metal incorporation brings the side advantage of increased optical absorption; from the sensitivity standpoint, this advantage can offset the lack of chemical amplification. Indeed, absorption at EUV is entirely dictated by elemental composition. The most promising elements for incorporation in a photoresist are those transition metals, and metals or semimetals with a large atomic number whose large $d$ orbitals result in considerable interaction cross section with EUV photons. These considerations arise from the observation of the atomic absorption cross sections of elements (reported in Figure 1 from tabulated data ${ }^{7}$ ). Notably, very few early experimental measurement of absorption have been previously reported at $\mathrm{EUV}^{8}$. In those works, transmissivity was found to be affected by the amount of incorporated tellurium. Experimental measurement of absorption in thin films is challenging for two main reasons. Primarily, a bright EUV source needs to be available to expose the material under conditions that are similar to those of actual scanner tool. Second, the photoresist needs to be tested in spin coated form as in the actual application. Because EUV photons are strongly absorbed by matter, only thin nitride membranes can be used for this purpose. Finally, the extraction of $\alpha$ requires the accurate measurement of the thickness of the coated film, which can only be done by an optical method (due to the fragility of the membrane).

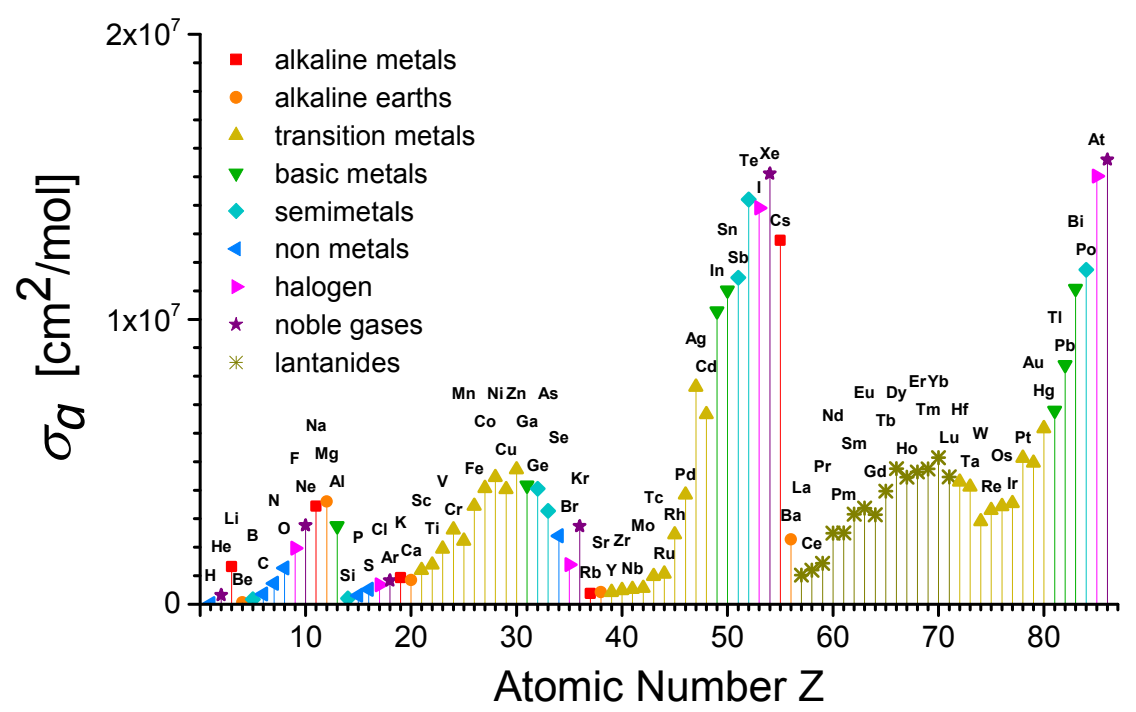

Figure 1. Atomic absorption cross-section $\sigma_{\mathrm{a}}$ at $\operatorname{EUV}(\lambda=13.5 \mathrm{~nm})$ of elements with atomic number $Z$ from 1 to 86 , from tabulated data ${ }^{7}$.

As an extension of our recent work $^{9}$, we here present the measurement of the linear absorption coefficient of metal-based photoresists in order to gain more insight into underlying mechanisms of the EUV resists. The methodology exploits the bright synchrotron light and the accurate estimation of all sources of uncertainty in the measurement. In the second part of the manuscript, the study of the exposure kinetics is presented, based on the bleaching rate. The study of the $\alpha$ and of the dose to clear can be combined to estimate the clearing volume of a photoresist and the effect of photoacid concentration in the microscopic quantities, according to previous models ${ }^{10}$.

\section{EXPERIMENTAL DETAILS}

\subsection{Transmittance measurement at the XIL beamline}

The XIL beamline at Swiss Light Source (SLS) features EUV light at $13.5 \mathrm{~nm}$ wavelength from a synchrotron source with average flux more than $30 \mathrm{~mW} / \mathrm{cm}^{2}$. The beamline is mainly used for EUV interference lithography ${ }^{11}$ and actinic mask inspection ${ }^{12}$. The beam intensity and shape is set by using a round pinhole of $30 \mu \mathrm{m}$ diameter. A square openframe mask $\left(0.5 \times 0.5 \mathrm{~mm}^{2}\right)$ is located in front of the sample to crop out the beam tail. The combination of pinhole and open-frame results in a highly homogenous beam intensity within the exposed area. A dedicated experimental setup was developed to measure the intensity of the light passing through a thin film in transmission mode and as a function of time as shown schematically in Figure 2. A photodiode is located behind the sample, and the photocurrent is collected at a high-speed acquisition $(50 \mathrm{~Hz})$. Because the membrane itself absorbs EUV light, the net flux $I_{0}$ is calibrated by 
measuring the transmittance of a blank silicon nitride membrane. Finally, the transmittance $T_{X}$ of the thin photoresist film is given by the ratio between measured photocurrent $I$ and the reference photocurrent $I_{0}$.

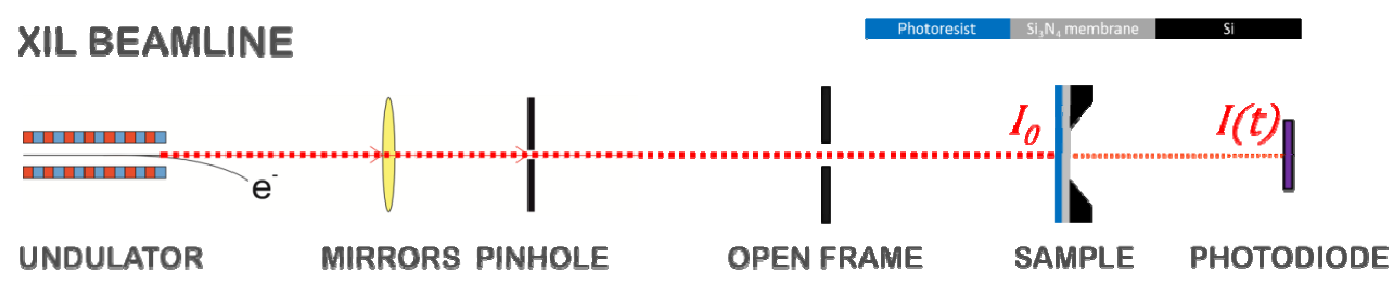

Figure 2. Schematic of experimental setup for absorption measurement at the XIL beamline.

\subsection{Linear Absorption Coefficient and Dill Parameters}

As the Beer-Lambert law describes, the amount of light transmitted through a homogeneous medium is a function of the thickness $d$ and of the linear absorption coefficient $\alpha$ :

$$
T_{X}=e^{-\alpha d} \mathrm{~T}_{\mathrm{X}}=\mathrm{e}^{-\alpha \mathrm{d}}
$$

From the previous equation, the linear absorption coefficient of the pristine, unexposed material can be calculated as:

$$
\alpha=-\frac{1}{d} \ln \frac{I\left(t_{0}\right)}{I_{0}}\left[\mu m^{-1}\right]
$$

where $I_{0}$ is the photocurrent measured through a blank membrane and $I\left(t_{0}\right)$ is the photocurrent thought the photoresist/membrane stack at the beginning of the exposure $\left(t=t_{0}\right)$. In our experimental setup, the current measured from the photodiode is recorded as a function of time, from the beginning and throughout the end of the exposure $\left(t=t_{\text {exp }}\right)$. Here, $t_{\text {exp }}$ is the time needed to fully expose the resist, calculated as the ratio between flux (which is constant during the exposure) and the dose-to-clear, which is also measured with the same tool a priori. We thus calculate the bleaching of the photoresist using the definitions of Dill parameters ${ }^{1}$ for the bleachable absorption coefficient $A$ :

$$
A=\frac{1}{d} \ln \frac{I\left(t_{\text {exp }}\right)}{I\left(t_{0}\right)}\left[\mu m^{-1}\right]
$$

the unbleachable absorption coefficient $B$ :

$$
B=-\frac{1}{d} \ln \frac{I\left(t_{\text {exp }}\right)}{I_{0}}=\alpha-A\left[\mu m^{-1}\right]
$$

and the exposure rate constant $C$ :

$$
C=\left.\frac{A+B}{A \Phi\left[I(0)-I(0)^{2} / I_{0}\right]} \frac{d I}{d t}\right|_{t=0}\left[\frac{c m^{2}}{m J}\right]
$$

In Eq. 5 the Dill parameter $\mathrm{C}$ has been reformulated as a function of the photocurrent measured with the diode, $I(t)$, rather than as a function of transmittance per unit energy $T_{X}(E)$, as in Dill's original definition. Because our measurement is carried out at constant EUV flux, the dose is constant per unit time and Eq. 5 is mathematically equivalent to the classical definition.

\subsection{Samples preparation and description}

Silicon nitride membranes $\left(100 \mathrm{~nm}\right.$ nominal thickness, $\left.3 \times 3 \mathrm{~mm}^{2}\right)$ suspended on a silicon frame $(280 \mu \mathrm{m}$ thick, $9 \times 9$ $\mathrm{mm}^{2}$ ), were fabricated by selective $\mathrm{KOH}$ etch of SiN-coated Si wafers. Thin photoresist films were subsequently spun coated on each membrane and processed according to manufacturer's specifications for what concerns pretreatment, post application bake, underlayer, and so on.

For the sensitivity (dosage curve) measurement, the CAR resists were spin coated on bulk silicon wafers of $500 \mu \mathrm{m}$ thickness, and processed according to manufacturer specifications. In this case, flood EUV exposures were carried out at increasing dose steps and the remaining resist thickness after development was measured with a stylus profiler (Dektak) to determine the dose to clear at $50 \%$ thickness. 
Non-chemically amplified metal-based photoresists were synthetized at the Advanced Research Center for Nanolithography (ARCNL). For applications in EUV lithography, metal containing materials offer the advantage of increased absorption at this wavelength in order to boost the sensitivity and balance the lack of chemical amplification. Sn cages, with five different counterions, were functionalized as negative-tone photoresists for EUV, to the purpose of achieving high absorbance and high etch resistance. The chemical formula of the basic building blocks was: $\left[\left(\mathrm{C}_{4} \mathrm{H}_{9} \mathrm{Sn}\right)_{12} \mathrm{O}_{14}(\mathrm{OH})_{6}\right] \mathrm{X}_{2}$, and schematically shown in Figure 3. The Sn cages were prepared as previously described in the literature ${ }^{13,14,15}$. The counterions $\mathrm{X}$ were: tosylate $\mathrm{CH}_{3} \mathrm{C}_{6} \mathrm{H}_{4} \mathrm{SO}_{3}{ }^{-}(\mathrm{Sn}-\mathrm{S})$, hydroxide $\mathrm{OH}^{-}(\mathrm{Sn}-\mathrm{OH})$, acetate $\mathrm{CH}_{3} \mathrm{COO}^{-}$ (Sn-A), malonate $\mathrm{CH}_{2}(\mathrm{COO})_{2}{ }^{2-}$ (Sn-M), trifluoroacetate $\mathrm{CF}_{3} \mathrm{COO}^{-}(\mathrm{Sn}-\mathrm{F})$. It is interesting to note that Sn-F contains fluorine and should, therefore, have a slightly higher absorbance. The patterning performance of these materials at EUV have been recently studied and show a good potential for high resolution and high sensitivity ${ }^{16}$. In addition, the chemical changes upon DUV exposure were studied for these materials and a chemical mechanism for the observed changes was proposed $^{17}$.



Figure 3. Schematic of tin cages structures. The counterions are indicated by the $\mathrm{X}$ (see text for detailed description).

One commercial zirconium oxocluster (ZrM1) was purchased from Sigma-Aldrich (CAS 189028-53-3) and used as is aside from the dilution rate which was tuned. A different zirconium (ZrM2) and a hafnium (HfM) methacrylate oxoclusters were prepared as described in previous works ${ }^{18,19}$. In this latter case, $\mathrm{Zr}(\mathrm{OiPr})_{4}$ and $\mathrm{Hf}(\mathrm{OBu})_{4}$ were mixed with methacrylic acid in acetonitrile and kept at room temperature. The formed crystals where filtered off and washed with acetonitrile. The structure of the compounds was previously deduced from thermogravimetric analyses, NMR and IR spectroscopy and XRD experiments reported elsewhere ${ }^{18,19}$. The molecular structure of the commercial compound has been previously elucidated by single crystal X-ray diffraction. Schematic structures of the ZrM2 and HfM are shown in the following Figure 4. For all the aforementioned metal based materials, the theoretical absorption was determined by the elemental composition and from the estimation of the density of the spin coated films based on the crystalline structures and accounting for the packing ratio.
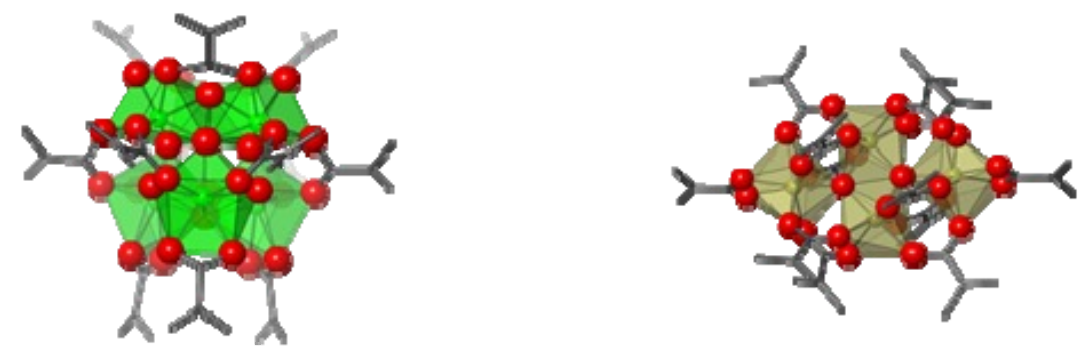

Figure 4. Schematic of Zr-based (left) and Hf-based (right) oxoclusters structures. Green (left) or brown (right) polyhedron delimits the coordination geometry of $\mathrm{Zr}$ and $\mathrm{Hf}$ atoms, respectively. Red balls represent oxygen atoms and gray bars represent carbon-carbon bonds. 
The Dill parameter $\mathrm{C}$ was measured in seven different formulations of a state-of-the-art chemically amplified EUV resist. The baseline system, P1_PAG1, used a standard polymer and standard photoacid generator (PAG) concentration. Two variant contained increased PAG loading of $120 \%$ and $140 \%$ of the baseline. The same backbone polymer was also synthesized with a different PAG and two different loadings (P1_PAG2 100\% and P1_PAG2 120\%). Finally, two alternative backbone polymers were formulated with the same baseline PAG and standard concentration (P2_PAG1 and P3_PAG1). All of these resists were organic. The samples description is provided in the following Table 1.

Table 1. Samples description, manufacturer and type.

\begin{tabular}{|l|l|l|l|l|}
\hline Resist & Manufacturer & Type & Chemically amplified & Metal \\
\hline Sn-M & ARCNL & condensed metal oxide & no & Sn \\
\hline Sn-A & ARCNL & condensed metal oxide & no & Sn \\
\hline Sn-F & ARCNL & condensed metal oxide & no & Sn \\
\hline Sn-S & ARCNL & condensed metal oxide & no & Sn \\
\hline Sn-OH & ARCNL & condensed metal oxide & no & Sn \\
\hline ZrM1 & Sigma-Aldrich & condensed metal oxide & no & Zr \\
\hline ZrM2 & ARCNL & condensed metal oxide & no & Zr \\
\hline HfM & ARCNL & condensed metal oxide & no & Hf \\
\hline P1_PAG1 & undisclosed & organic polymer & Yes & - \\
\hline P1_PAG2 & undisclosed & organic polymer & Yes & - \\
\hline P2_PAG1 & undisclosed & organic polymer & Yes & - \\
\hline P3_PAG1 & undisclosed & organic polymer & Yes & - \\
\hline
\end{tabular}

\subsection{Spectroscopic ellipsometry}

The accurate measurement of the thickness of resist coated on a suspended membrane is technically challenging and it is a major source of uncertainty in the estimation of $\alpha$. As a matter of fact, the coating thickness varies greatly depending on the substrate and further more on flexible membranes. Resist thickness was measured using a M-2000 spectroscopic ellipsometer from J.A. Woollam, equipped with a 75W Xe ARC light source, in the spectral range 250-1000 nm. The spot size was about $300 \mu \mathrm{m}$ in diameter. Thickness measurements were recorded on a $1 \mathrm{x} 1 \mathrm{~mm}^{2}$ area of the membrane, with a step size of $0.2 \mathrm{~mm}$. The ellipsometric data was modeled as two layers: for the $\mathrm{SiN}_{\mathrm{X}}$ membrane, using a $\mathrm{SiN}_{\mathrm{X}}$ (Cody-Lorentz) model and for the resist layer, using a Cauchy model. Initial material fits and optical constants of the $\mathrm{SiN}_{\mathrm{X}}$ membrane were generated by angle scan on the silicon frame surrounding the suspended membrane. For the resist layer, angle scans were measured on Si wafers coated only with the respective resist materials. Neither additional layers nor interfaces were needed to obtain a good fitting to the data. Furthermore, the same material was spin-coated and analyzed at several thickness ranges. This approach significantly reduces the uncertainty in the $\alpha$, using a combination of optical inspection and spectroscopic ellipsometry to estimate $d$ in the proximity of the exposed area.

\subsection{Estimation of uncertainty}

The uncertainty in the measurement of $\alpha$ arises from three sources. The first is the uncertainty in the measurement of the resist thickness $\left(\sigma_{d}\right)$, due to the non-uniformity of the coating. The second is the uncertainty in the thickness of the silicon nitride membrane, due to the $\mathrm{KOH}$ etch variability, which affects the value of the reference photocurrent $\left(\sigma_{I 0}\right)$. The third is the uncertainty in the measured photocurrent from the photodiode $\left(\sigma_{I}\right)$. The standard deviation of $\alpha$ is then given by the well-known formula for error propagation and replacing the due quantities: 


$$
\sigma_{\alpha}=\sqrt{\left(\frac{\partial \alpha}{\partial d}\right)^{2} \sigma_{d}^{2}+\left(\frac{\partial \alpha}{\partial I_{0}}\right)^{2} \sigma_{I_{0}}^{2}+\left(\frac{\partial \alpha}{\partial I(t)}\right)^{2} \sigma_{I(t)}^{2}} \cong \sqrt{\left(\frac{\partial \alpha}{\partial d}\right)^{2} \sigma_{d}^{2}+\left(\frac{\partial \alpha}{\partial I_{0}}\right)^{2} \sigma_{I_{0}}^{2}}=\sqrt{\alpha^{2} \frac{\sigma_{d}^{2}}{d^{2}}+\frac{1}{d^{2}} \frac{\sigma_{I_{0}}^{2}}{I_{0}^{2}}}
$$

From experimental data, the fluctuations in measured photocurrent were negligible in comparison to the other terms of Eq. 6 and, therefore, were not taken into account.

We prepared the samples by spin-coating each material to several thickness values. Provided that the logarithm of transmittance varies linearly with the film thickness, the conditions of homogeneous absorption is satisfied and Eq. 1 holds. When several thickness/transmittance data points are available, the slope of the linear fit to the data is used to determine the absorption coefficient $\alpha$. Each measured data point accounts for standard deviation along both the abscissa and the ordinates, which represent the uncertainty in transmittance $T_{X}$ and thickness $d$, respectively. The standard deviation of $\alpha$ is finally estimated as the standard deviation of the slope of the least-squares linear fit to the data with weighting on error bars along both $X$ and $Y$ axis, according to a numerical implementation of a method described elsewhere $^{20}$. In sum, this approach greatly reduces the uncertainty in the estimation of $\alpha$.

\section{ABSORPTION COEFFICIENT OF METAL-BASED PHOTORESISTS}

Photocondensed metal oxide resists have been coated at thickness ranging from 11 to $76 \mathrm{~nm}$, which are of relevance for applications in EUV lithography. The transmittance, $T_{X}$, is then plotted versus the measured thickness, $d$, as determined by spectroscopic ellipsometry in Fig. 5. Notably, all samples studied in this work exhibited a linear trend. It is thus appropriate to extract the $\alpha$ as the slope of the linear fit to the data (shown in the figure), according to the methodology described in the previous section.

The extracted $\alpha$ for the metal-containing resists are summarized in Figure 6 . As a matter of comparison, the absorption coefficient of a typical chemically amplified resist - purely organic - is also shown (gray bar). The absorption of tincontaining resists ranged from about 11 to $14.7 \mu^{-1}$, which is about two times or three times higher than conventional CAR resists based on an organic backbone, owing to the remarkably large absorption cross section of tin at EUV. $\alpha$ also shows some dependence on the counterion group. Contrary to expectations, the higher absorption of the fluorinecontaining Tin-F (trifluoroacetate counterions) compared to Tin-A (acetate counterions) was not observed experimentally. A reason could be a lower density of Tin-F compared to Tin-A due to, for instance, a small amount of solvent trapped in the film or different packing arrangement. Furthermore, the counterions constitute only a low fraction of the absorption considering the strongly absorbing Sn atoms; the theoretical absorption for Tin-F is $13.9 \mu \mathrm{m}^{-1}$ compared to $13.6 \mu^{-1}$ for Tin-A. Further study is envisioned to clarify how the counterion structure affects the packing density of the material after spin coating and, therefore, its effect on optical absorption. 
a)

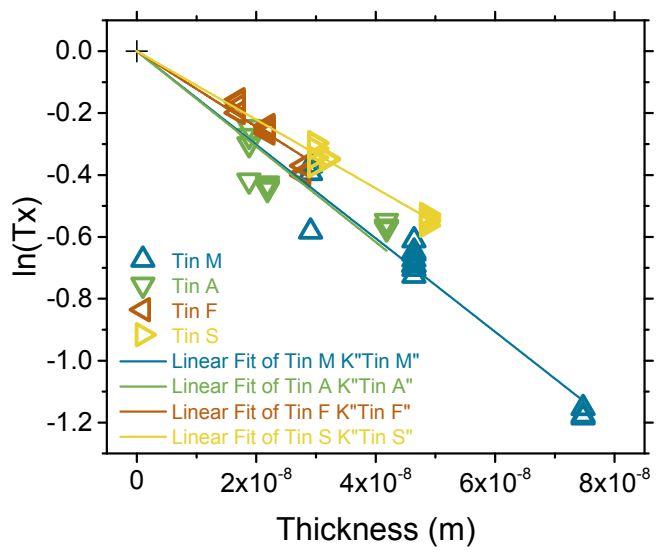

b)

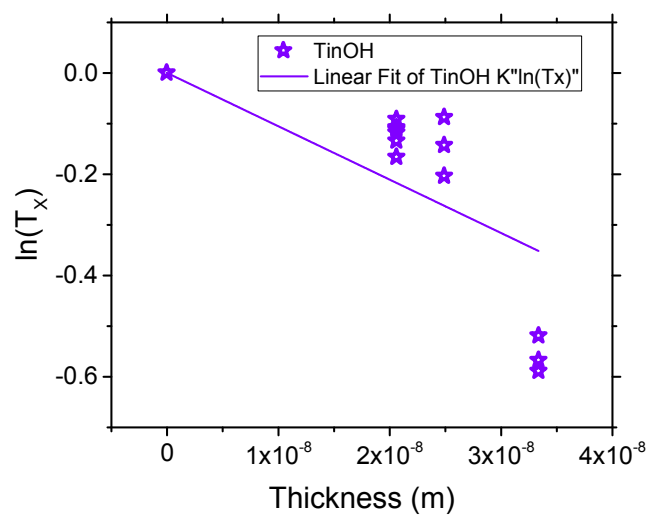

c)

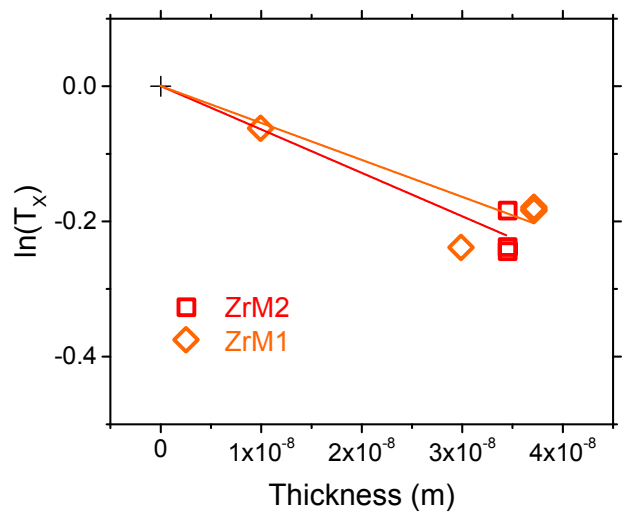

Figure 5. Logarithm of measured transmittance as a function of resist film thickness of photocondensed metal oxides based on tin cages (a)(b) and zirconium (c). The solid lines are the least-squares linear fit to the data.

The enhancement in EUV absorption was not as much significant in zirconium-containing compounds, where the absorption was only slightly higher than conventional CAR. This result is ascribed to the relatively small absorption cross section of $\mathrm{Zr}$ in comparison to oxygen and carbon, the main constituents of organic resists. There was not a sizeable difference, within experimental error, between the commercial compound (ZrM1) and the one prepared in house 
(ZrM2). As for the hafnium-based material, it showed about $9 \mu \mathrm{m}^{-1}$ absorption, which is due to the good absorptivity of the element, and to the remarkably higher density $(\approx+50 \%)$ than conventional organic resists. In the case of the HfM sample, only one thickness sample was available for measurement and, for this reason, no linear fit is shown in Fig. 5 and the associated error bar is significantly larger than all other samples.

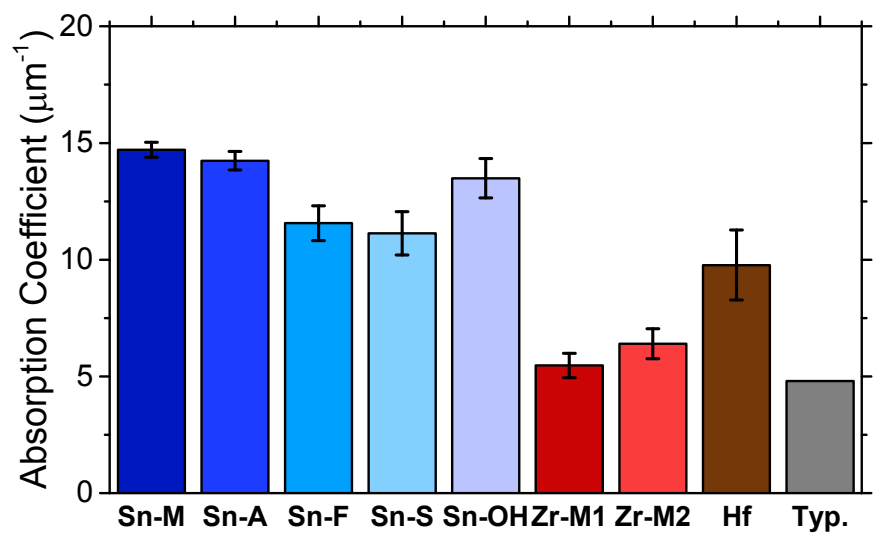

Figure 6. Summary of measured linear absorption coefficient $\alpha$ of photocondensed metal oxides: tin cages with different counterions (shades of blue), zirconium oxoclusters (shades of red) and hafnium oxocluster (brown). The uncertainty of $\alpha$ is shown as error bar. The absorption of a typical organic photoresist at EUV is also shown for comparison (gray bar).

The theoretical values of the absorption coefficient of these materials was estimated from elemental absorption cross section at EUV, weighted on the relative amount of each element. The density of the spin coated film was estimated from the crystalline structure of the individual molecule and taking into account the packing arrangement. The comparative plot in Fig. 7 shows the good agreement between experiment and estimate. For some of the tin-based compounds, the measured absorptivity was slightly lower than expected; the opposite trend was found for the $\mathrm{Hf}$ and $\mathrm{Zr}$ materials. As mentioned previously, differences in the actual density of the film after spin coating (for example, due to solvent trapping in the film) can affect the measured value too. In summary, the actual absorption can be substantially different from the expected value, and thus affect the resulting lithographic sensitivity and performance of the photoresist.

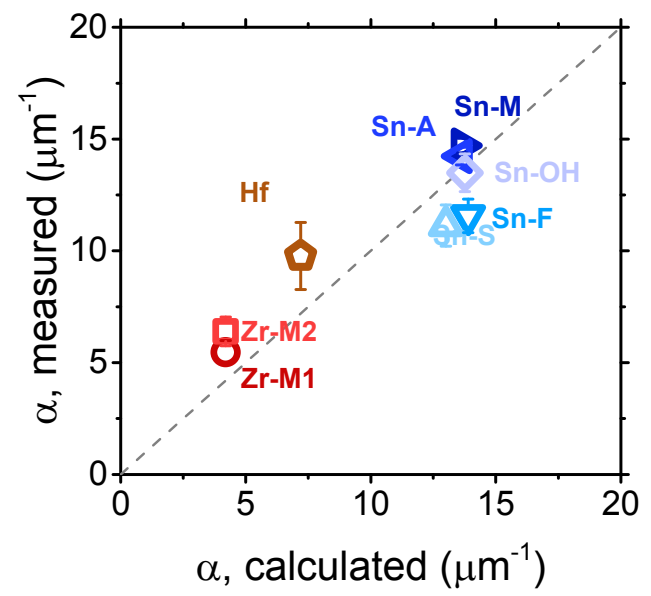

Figure 7. Comparison of measured linear absorption coefficient $\alpha$ versus estimated $\alpha$ for metal-based photoresists synthesized at ARCNL. The matching between experiment and calculations is quite good. The discrepancy can be ascribed to differences in the density of the material after spin coating, for instance due to incomplete evaporation of the solvent of due to packing arrangements of the basic chemical blocks. 


\section{DILL PARAMETER C OF CHEMICALLY AMPLIFIED RESISTS}

The Dill parameter $\mathrm{C}$ defines the rate of bleaching of a photoresist at the beginning of the exposure. Using our experimental setup we are able to simultaneously measure the transmittance change while exposing the material to EUV radiation. Furthermore, the incident flux is intense enough to cause a sizeable bleaching (about $1 / 10^{\text {th }}$ of the flux density of an actual EUV scanner tool). In DUV lithography, the Dill parameter $\mathrm{C}$ was associated with the reaction kinetics and the rate of photoacid generation from radiation-induced dissociation of a photoactive compound in chemically amplified resists. In the case of exposure to monochromatic light, the kinetics was found to be described by a first order kinetics where the PAG amount is exponentially decaying with a rate equal to the product between Dill C and incident flux. This model also provides an analytical expression for the amount of photoacids generated per absorbed photon, also known as the quantum yield $(\mathrm{QY})^{21}$.

Selected CARs specifically designed for EUV lithography from undisclosed manufacturers have been exposed and the rate of bleaching has been measured. Difficulty in accurately measuring Dill $\mathrm{C}$ lies primarily in detecting a small variation in the photocurrent (due to the bleaching) out of a large common mode signal (due to the intense transmitted flux, $\approx 80 \%$ of the total incident flux). This observation is a direct consequence of the small bleaching of EUV photoresists as compared to pre-EUV photoresists ${ }^{9}$. Besides, the measured photocurrent is affected by large electronic noise due to the fast acquisition time $(50 \mathrm{~Hz}$ in our case), which does not allow for smoothing over a large integration time. As a consequence, the linear fitting of the slope of the data set affected by significant noise with only about 20 data points per unit time results in large uncertainty. Previous experimental measurement of the Dill parameter C at DUV could rely on the large bleaching and, therefore, large signal to noise ratio. At EUV, methods include the estimation of outgassing rate by flooding electron beam exposure ${ }^{22}$, Fourier transform infrared spectroscopy ${ }^{23}$, and by titration methods $^{24}$.

The scope of this experiment is to detect any effect of: 1) the PAG loading, 2) the PAG molecule ionizability, and 3) the polymer backbone ionizability on the Dill parameter C. The set of samples, described in Section 2, makes it possible to discern each of these effects. Rather than preparing a thickness range of photoresists (which would have been not possible while maintaining the same chemical properties), this set of CARs was prepared by tuning the spin coating speed to target $30 \mathrm{~nm}$ for all samples, according to manufacturer's specifications.

Preliminarily, the absorption coefficient of these materials was measured. As shown in Figure 8, the $\alpha$ was, within experimental error, basically unaffected by the formulation which can be attributed to the lack of changes in density and elemental composition.

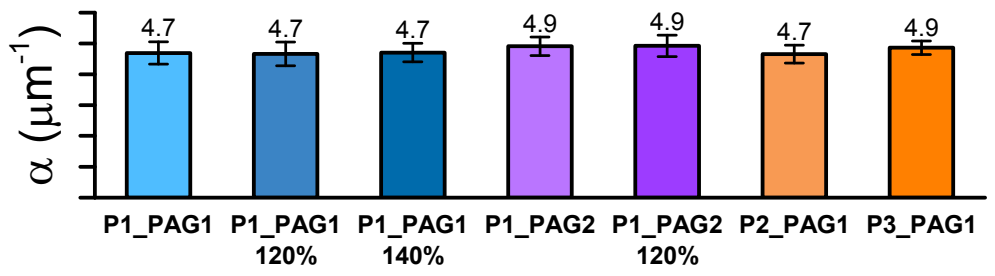

Figure 8. The absorption coefficients of the set of CARs described in Section 2.

The measurement of the bleaching rate (Figure 9) shows a sizeable effect of the PAG molecule on the Dill parameter C. The PAG2 molecule is more reactive and more easily ionizable than molecule PAG1, which results in about $50 \%$ larger Dill $\mathrm{C}$ or, in other words, ionization cross section. The effect of PAG loading was instead negligible in both PAG. Previous studies have found a dependence of Dill C on the PAG loading only at high concentrations and anyway with a nonlinear dependence $\mathrm{e}^{25,26}$. Elsewhere, it was found that the PAG loading only affects the lithographic sensitivity but not the Dill $\mathrm{C}^{27}$. It is not possible to clearly state where, on the Dill C vs. PAG loading curve, our baseline is located. As a result, we can only conclude that, up to $+40 \%$ PAG loading, a typical CAR resist exhibits no increase in bleaching rate. It should be noted however that heavy loadings are not feasible in practical CAR formulations due to other competing effects. 


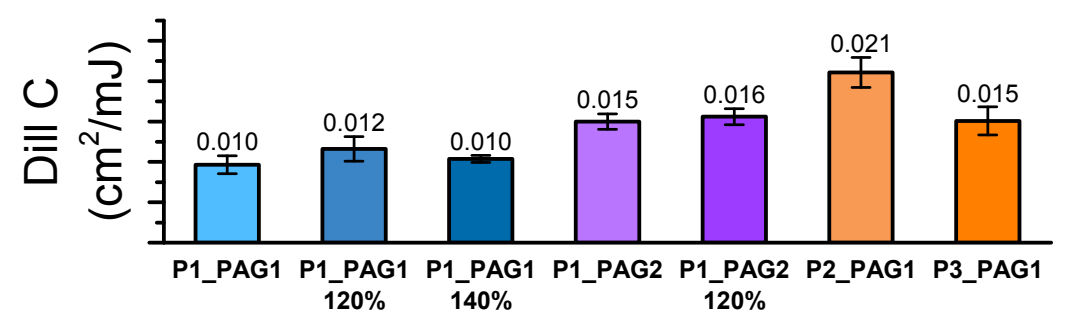

Figure 9. The Dill parameters C of the set of CARs described in Section 2.

Interestingly, the backbone polymer also plays a role in the rate of photoacid generation. A change in backbone can result in up to twice as larger Dill parameter, as detected in the case of Polymer 2, all the other PAG features being the same as the baseline. In the scope of this work we again assume that all PAG kinetics is completely described by the bleaching measured during EUV exposure. These observations show that the Dill C can be tuned in a variety of ways by the manufacturer, and not only by acting on the PAG as one might expect.

\section{CONCLUSIONS}

The absorption coefficient at EUV of photocondensed metal oxides has been measured experimentally using a dedicated tool and synchrotron light source. By preparation of a series of thickness, the $\alpha$ was measured with unprecedented accuracy. It was found that tin containing compounds have twice or three times higher absorption than conventional chemically amplified resists based on organic polymers. Zirconium materials showed a slightly higher absorption, which we ascribe to the low contribution of $\mathrm{Zr}$ to the overall absorption cross section of the material. Notably, the actual absorption $\alpha$ can significantly differ from the calculated theoretical value. As a result, the lithographic performance and the sensitivity should also be examined in the light of the real absorptivity.

The study of the bleaching rate reveals that the Dill parameter $\mathrm{C}$ can be tuned by changing not only the PAG molecule but the polymer backbone as well. However, we did not detect any effect of PAG loading up to $+40 \%$ of the baseline, within experimental error. The resulting ionization cross section was found to increase by up to twice as much as the baseline formulation. Under the assumption that the bleaching fully describes the reaction kinetics, the rate of PAG formation strongly depends on the PAG ionizability. It is important to remark that this latter assumption is not fully valid under the exposure conditions of EUV, where other physical phenomena can and do contribute to absorption and secondary electron generation, but not to photoacid generation. Nevertheless, these preliminary results lead us to envisage further investigations to quantitatively estimate these effect from an experimental point of view.

\section{ACKNOWLEDGEMENTS}

The beamline support from Michaela Vockenhuber and Markus Kropf (PSI) is kindly acknowledged. We also wish to thank Iacopo Mochi (PSI) and Jason Stowers (Inpria) for fruitful discussions. Spectroscopic ellipsometry has been carried out with the technical support of Goran Milinkovic and Pieter-Jan van Zwol (ASML).

\section{REFERENCES}

${ }^{1}$ Dill, F. H., Hornberger, W. P., Hauge, P. S., Shaw, J. M., IEEE Transactions On Electron Devices 22(7), 445 (1975).

${ }^{2}$ Kozawa, T. and Tagawa, S., Japanese Journal of Applied Physics 49, 030001 (2010).

${ }^{3}$ Zanchetta, E. Della Giustina, G., Grenci, G., Pozzato, A., Tormen, M., and Brusatin, G., Adv. Mater. 25, 6261-6265 (2013).

${ }^{4}$ Chakrabarty, S., Ouyang, C., Krysak, M., Trikeriotis, M., Cho, K., Giannelis, E. P., Ober, C. K., "Oxide nanoparticle EUV resists: toward understanding the mechanism of positive and negative tone patterning”, Proc. SPIE 8679, 867906 (2013). 
${ }^{5}$ De Simone, D., Sayan, S., Dei S., Pollentier, I. Kuwahara, Y., Vandenberghe, G., Nafus, K., Shiratani, M., Nakagawa, H., Naruoka, T., Proc. SPIE 9776, 977606 (2016).

${ }^{6}$ Stowers, J., Anderson, J., Cardineau, B., Clark, B., De Schepper, P., Edson, J., Greer, M., Jiang, K., Kocsis, M., Meyers, S., Telecky, A., Grenville, A., De Simone, D., Gillijns, W., Vandenberghe, G., Proc. SPIE 9779, 977904 (2016)

${ }^{7}$ Henke, B. L., Gullikson, E. M., and Davis, J. C., "X-ray interactions: photoabsorption, scattering, transmission, and reflection at $\mathrm{E}=50-30000 \mathrm{eV}, \mathrm{Z}=1-92$," Atomic Data and Nuclear Data Tables 54(2), 181-342 (1993).

${ }^{8}$ Sekiguchi, A., Matsumoto, Y., Harada, T., Watanabe, T., and Kinoshita, H., Proc. of SPIE Vol. 9422 94222L-2 (2015)

${ }^{9}$ Fallica, R., Stowers, J. K., Grenville, A., Frommhold, A., Robinson, A. P. G., and Ekinci, Y., "Dynamic absorption coefficients of chemically amplified resists and nonchemically amplified resists at extreme ultraviolet", J. Micro/Nanolith. MEMS MOEMS 15(3), 033506 (2016).

${ }^{10}$ Mack, C. A., Thackeray, J. W., Biafore, J. J., Smith, M. D., J. Micro/Nanolith. MEMS MOEMS 10(3), 033019 (2011).

${ }^{11}$ Mojarad, N., Hojeij, M., Wang, L., Gobrecht J. and Ekinci, Y., "Single-digit-resolution nanopatterning with extreme ultraviolet light for the $2.5 \mathrm{~nm}$ technology node and beyond", Nanoscale 7, 4031-4037 (2015)

${ }^{12}$ Helfenstein, P., Mohacsi, I., Rajeev, R., Ekinci, Y., "Scanning coherent diffractive imaging methods for actinic extreme ultraviolet mask metrology", J. Micro/Nanolith. MEMS MOEMS 15(3), 034006 (2016).

${ }^{13}$ Eychenne-Baron, C., Ribot, F., and Sanchez, C., "New synthesis of the nanobuilding block [(BuSn)12O14(OH)6]2+ and exchange properties of $[(\mathrm{BuSn}) 12 \mathrm{O} 14(\mathrm{OH}) 6](\mathrm{O} 3 \mathrm{SC} 6 \mathrm{H} 4 \mathrm{CH} 3) 2$ ", Journal of Organometallic Chemistry 567(1-2), 137-142 (1998).

${ }^{14}$ van Lokeren, L., Willem, R., van der Beek, D., Davidson, P., Morris, G.A., Ribot, F., "Probing the anions mediated associative behavior of tin-12 oxo-macrocations by pulsed field gradient NMR spectroscopy”, J. Phys. Chem. 114, 16087-16091 (2010)

${ }^{15}$ Cardineau, B., Del Re, R., Marnell, M., Al-Mashat, H., Vockenhuber, M., Ekinci, Y., Sarma, C., Freedman, D.A., Brainard, R.L., "Photolithographic properties of tin-oxo clusters using extreme ultraviolet light (13.5 nm)", Microelectr. Eng. 127, 44-50 (2014).

${ }^{16}$ Haitjema, J., Zhang, Y., Vockenhüber, M., Kazazis, D., Ekinci, Y., Brouwer, A.M., “Tin-oxo cages for use as photoresists in EUV lithography”, Proc. SPIE 10143-72 (2017).

${ }^{17}$ Zhang, Y., Haitjema, J., Liu, X., Lindblad, A., Castellanos, S., Ottosson, N., Brouwer, A.M., "Photochemical conversion of a tin-oxo cage compound studied using hard x-ray photoelectron spectroscopy", Proc. of SPIE 10146-5, (2017).

${ }^{18}$ Kickelbick, G. and Schubert, U., Chem. Berich. 130, 473-477 (1997)

${ }^{19}$ Gross, S., Kickelbick, G., Puchberger, M., and Schubert, U., Monatshef. Chem. 134, 1053-1063 (2003)

${ }^{20}$ York, D., Evensen, N., Martinez, M. and Delgado J., "Unified equations for the slope, intercept, and standard errors of the best straight line", Am. J. Phys. 72(3), 367 (2004)

${ }^{21}$ Mack, C. A., "Absorption and exposure in positive photoresist”, Appl. Optics 27(23), 4913 (1988).

${ }^{22}$ Hassanein, E., Higgins, C., Naulleau, P., Matyi, R., Gallatin, G., Denbeaux G., et al. "Film quantum yields of EUV and ultra-high PAG photoresists", Proc SPIE Vol. 6921, 69211I (2008).

${ }^{23}$ Fedynyshyn, T. H., Goodman, R. B., Cabral, A., Tarrio C., and Lucatorto, T. B., "Polymer Photochemistry at the EUV Wavelength“, Proc. of SPIE Vol. 7639, 76390A (2010).

${ }^{24}$ Szmanda, C. R., Brainard, R. L., Mackevich, J. F., Awaji, A., Tanaka, T., and Yamada, Y., Bohland, J., Tedesco, S., Dal'Zotto, B., Bruenger W., Torkler, M., Fallmann, W., Loeschner, H., Kaesmaier, R., Nealey, P. M., and Pawloski, A. R., "Measuring acid generation efficiency in chemically amplified resists with all three beams", J. Vac. Sci. Technol. B 17(6), 3356 (1999).

${ }^{25}$ Higgins, C. D., Szmanda, C. R., Antohe, A., Denbeaux, G., Georger, J., and Brainard, R. L., "Resolution, Line-Edge Roughness, Sensitivity Tradeoff, and Quantum Yield of High Photo Acid Generator Resists for Extreme Ultraviolet Lithography", Jpn. J. Appl. Phys. 50, 036504 (2011)

${ }^{26}$ Hirose, R., Kozawa, T., Tagawa, S., Kai, T., and Shimokawa, T., "Dependence of Acid Generation Efficiency on Molecular Structures of Acid Generators upon Exposure to Extreme Ultraviolet Radiation", Appl. Phys. Express 1, 027004 (2008).

${ }^{27}$ Gronheid, R., Vaglio Pret, A., Rathsack, B., Hooge, J., Scheer, S., Nafus, K., Shite, H., Kitano, J., "Resolutionlinewidth roughness-sensitivity performance tradeoffs for an extreme ultraviolet polymer bound photo-acid generator resist“, J. Micro/Nanolith. MEMS MOEMS 10(1), 013017 (2011) 